\title{
Calculated and simulated effects of heterogeneous dose distributions in radiotherapy using the dose volume inhomogeneity corrected biological equivalent dose formula with special reference to prostate cancer
}

\author{
BO LENNERNÄS ${ }^{1}$, PER ALBERTSSON $^{1}$, MALIHA EDGREN $^{2}$ and STEN NILSSON ${ }^{2}$ \\ ${ }^{1}$ Department of Oncology, Sahlgrenska University Hospital, SE 41345 Gothenburg; \\ ${ }^{2}$ Department of Oncology, Karolinska University Hospital Solna, SE 17176 Stockholm, Sweden
}

Received July 27, 2007; Accepted September 7, 2007

\begin{abstract}
The aim of this study was to evaluate and estimate the impact on the biological effective dose (BED) of irradiation delivered to a tumour during high dose rate brachytherapy with a heterogeneous dose distribution in the target volume. The calculation of BED in combination with the critical-voxel model and the LQ (linear quadratic) model was used to evaluate the effect of different combinations of heterogeneous dose distribution. The model is called the dose volume inhomogeneity corrected BED (DVIC-BED). Different random and non-random combinations of radiobiological parameters were used to test the model. A simulated clinical treatment of two 10 Gy fractions of brachytherapy was used. In the simulations $0-100 \%$ of the target volume was simulated to receive only $80 \%$ of the total dose. Different $\alpha / \beta$ ratios as well as a different $\alpha$ value were used. A drastic effect on the outcome was observed especially when the ratio was low and the $\alpha$ value was high. The clinical effect is amplified when the tumour is considered to have a step dose respond curve. A 5 Gy decrease in dose corresponds to a reduction in clinical or chemical control $\leq 10-25 \%$ in the interval 65-85 Gy. Random production of different values has basically the same effect as above. The result stresses the importance to have control of the dose and the target volume during brachytherapy of prostate cancer. This is even more important when monotherapy with high dose rate brachytherapy is used and with a low $\alpha / \beta$ ratio. The advantage of using this formula is that it is based on the LQ/ BED formula and that different treatments with different fractions and treatments can be
\end{abstract}

Correspondence to: Dr Bo Lennernäs, Department of Oncology, Sahlgrenska University Hospital, SE 41345 Gothenburg, Sweden E-mail: bo.lennernas@telia.com

Key words: dose volume inhomogeneity corrected biological effective dose, linear quadratic, four-dimensional, prostate cancer, radiotherapy summated independently of the homogeneity of the dose distribution.

\section{Introduction}

Prostate cancer PC is the most common malignancy that afflicts Swedish men. Every year, 10000 new cases are diagnosed (1). Radiotherapy is an established method for the curative treatment of PC (2-4). Local control seems to be an important factor as regards survival and Hanks and coworkers have presented dose-response curves for PC (5).

In Sweden a 192-Irridium after-loading system is used for the treatment of PC and this technique has now been implemented in several centres and $>2000$ patients have been treated with the technique $(4,6)$. External beam radiotherapy is developing from three-dimensional (3D) to fourdimensional (4D) in order do minimize different errors in patient/target positioning (7), and intensity modulated radiotherapy (IMRT) is also a well established method for treating PAC. All these methods will, due to technical reasons, result in a heterogeneous dose distribution.

The linear quadratic (LQ) and the extension, the biological equivalent dose (BED), formulas are well suited for tumour control probability (TCP) calculations, but LQ-formula is not well suited for treatments with heterogeneous dose distributions. However, Lennernäs and Nilsson (8) have presented the dose volume inhomogeneity corrected BED (DVICBED) for TCP calculations in evaluation of external radiation treatments with certain displacement errors. This formula can be used in BED calculations in treatments with a heterogeneous dose-distribution such as brachytherapy, IMRT or the summated dose of 4D external beam treatments.

In this study, we present DVIC-BED with special reference to different $\alpha / \beta$ ratios and heterogeneous dose distributions in radiotherapy of prostate cancer.

\section{Materials and methods}

Calculation model. The principle of the dose volume inhomogenity corrected biological equivalent dose (DVIC-BED) is to calculate the biological effect in several subvolumes of 
the target and then to add these sub-effects and express the sum as a dose as if the target had received this uniform dose.

The DVIC-BED model is a combination of linear-quadratic (LQ)-formula and the principle of the critical volume (tissuerescuing unit) model $(9,10)$. The principle has been presented elsewhere (8) and is summarised below.

If the number of clonogenic cells is $c$ ( $c_{t}$ at specific time), the number of fractions is $\mathrm{n}$, fraction dose $\mathrm{d}$, total dose $\mathrm{D}$ and survival fraction SF and E for the effect after several fractions, the following expression for tumour control probability (TCP) can be derived (10):

$\mathrm{TCP}=\mathrm{e}^{-\mathrm{c}_{\mathrm{t}}}$

$c_{t}=c_{0} \times S F$

$\left.\mathrm{SF}=\mathrm{e}^{(-\alpha \mathrm{D}-} \beta \mathrm{dD}\right)$

$E=-\ln \left(e^{(-\alpha D-} \beta^{d D}\right)$

If one assumes that the clonogenic cells are homogeneously distributed in the tumour from the beginning, one can divide the tumour into several subvolumes (v). The probability for cure will then depend on the number of clonogenic cells in each volume according to (10):

$\left.\mathrm{TCP}=\mathrm{e}^{-\left(\left(\mathrm{c}_{0} / \mathrm{v} \times \mathrm{e}-\mathrm{E}_{1}\right)\right.}+\ldots . .+\left(\mathrm{c}_{0} / \mathrm{v} \times \mathrm{e}^{-\mathrm{E}_{\mathrm{V}}} \mathrm{v}\right)\right)=$

$=\mathrm{e}-\left(\mathrm{c}_{0} / \mathrm{v} \times\left(\mathrm{e}^{-\mathrm{E}} 1+\ldots .+\mathrm{e}^{-\mathrm{E}_{\mathrm{V}}}\right)\right)$

The disadvantage is that the clonogenic number is unknown and the equation can only be solved using clinical data. The LQ formula, on the other hand, does not include the number of clonogenic cells, but instead expresses the relative effect on the tumour or other tissue.

In 1989, Fowler introduced the concept of the biologically effective dose and this is expressed as (11):

$\mathrm{BED}=\mathrm{E} / \alpha=\mathrm{D}(1+\mathrm{d} /(\alpha / \beta))$

The disadvantage of LQ is that the calculation assumes a homogeneous dose distribution and, as above, only can calculate the relative effect. The difference between relative and absolute effect is obvious in LQ-calculations on T1 and T3 PAC tumours - the LQ formula makes no difference. It is the clinical parameters that determine the total dose, whether the LQ-formula can analyse different fractionation schedules using that dose.

In clinical practice calculations of the relative effect is an effective tool, but when using intensity modulated radiation therapy (IMRT) or brachytherapy the limitations of a homogeneus dose is clear. The advantage of using the LQformula is that it contains the $\alpha / \beta$ ratio (which reflect the radiobiological properties) and that BEDs from different treatments can be added. The BED calculation assumes that complete repair occurs between two consecutive fractions. It contains no correction for the cell proliferation which might occur during treatment. This is acceptable in the case of the late reacting tissue and in PAC since most studies show no correlation between treatment times on local control (12-14), when the treatment time is $<8$ weeks.
In order to calculate the BED in a target with an inhomogeneous dose distribution it is possible to combine equation 4 and 5 to the expression:

$\mathrm{BED}=-\left(\ln \left(\mathrm{e}^{-\mathrm{E}} 1+\mathrm{e}^{-\mathrm{E}} 2+\ldots \mathrm{e}^{-\mathrm{E}} \mathrm{Vn} / \mathrm{vn}\right)\right) / \alpha$

The advantage of using this formula is that it is based on the $\mathrm{LQ} / \mathrm{BED}$-formula and that different treatments with different fractions and treatments can be summated independently of the homogeneity of the dose distribution. The principle of DVIC-BED is to estimate and summate on a known number of subvolumes instead of calculating the effect on each clonogenic cell - a number which is not known.

Treatment. The treatments simulated in this study is a combination of 50 Gy external radiotherapy given in 2 Gy fractions in two 2.5 weeks with a split in two weeks, and two $10 \mathrm{~Gy}$ brachytherapy treatments given in the beginning and end of the split. However, only the effect of the brachytherapy part is presented.

The $\alpha / \beta$ ratio and $\alpha$-values have been published by Eklöv (4.14, $\alpha=0.12)$ for ${ }^{60} \mathrm{Co}$ and DeWeese et al $(3.7-10.9, \leq 0.064$ $0.115)$ for both high and low dose rate irradiation $(15,16)$. Although these values are determined in vitro, PAC can be assumed to have both a low $\alpha$ value and a low $\alpha / \beta$ ratio (Lennernäs). The ratio has been subject for discussion by Brenner, Hall and Fowler and the ratio presented was as low as $1.5(11-14)$.

The simulation is divided in several parts. First, the effect of dose-reduction in subvolumes of the target is discussed. In this part, the target volume is divided into 100 smaller volumes and these volumes are irradiated with different doses. The output of the calculations are recalculated and presented as if the treatments were given as external beam radiotherapy in 2 Gy fractions. In the second part, the $\alpha$ and $\alpha / \beta$ ratio is randomised $\sim 0.25$ and 5.75 , respectively.

Estimation of doses required for local control and for causing side effects. The dose required to achieve local control of prostate cancer has been suggested by Hanks et al (5). These curves are important in understanding the effect of the DVICBED calculations and are the ultimate clinical solutions of the equations presented above.

\section{Results}

As shown in Fig. 1A and B, under dosage of even small volumes of the target has a drastic effect on DVIC-BED and it depends mainly on the minimum dose delivered to any volume of the target if the ratio is low and the $\alpha$ is high.

Fig. 2A shows that all values $(\alpha, \beta$ and $\alpha / \beta$ ratio) are randomised in each treatment. The values are the same in all sub-volumes. Different combination will slightly influence the slope of the curve, but the initial step decrease in DVICBED is always present in the volume $0-20 \%$. Thus, small misses are important.

As shown in Fig. 2B, all values in all sub-volumes are randomised, and the effect is still drastic. The highest curve (A) represents a high ratio (5) and low $\alpha(0.2)$, the lowest curve (C) a low ratio (3) and high $\alpha(0.4)$ respectively and (B) the 
A

\section{DVIC-BED}

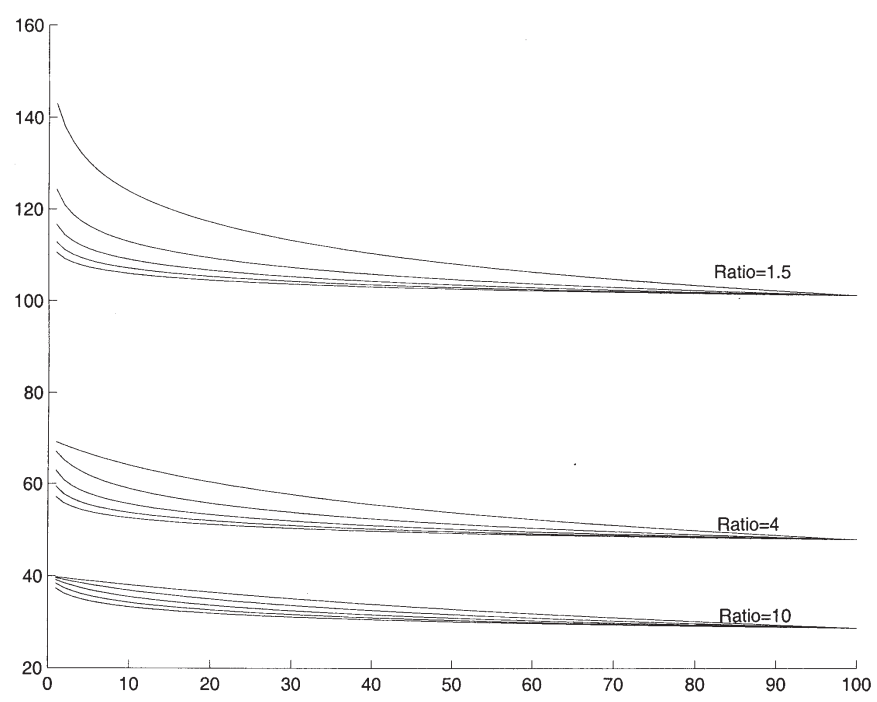

$\%$ Subvolume with underdosage

\section{B}

\section{DVIC-BED}

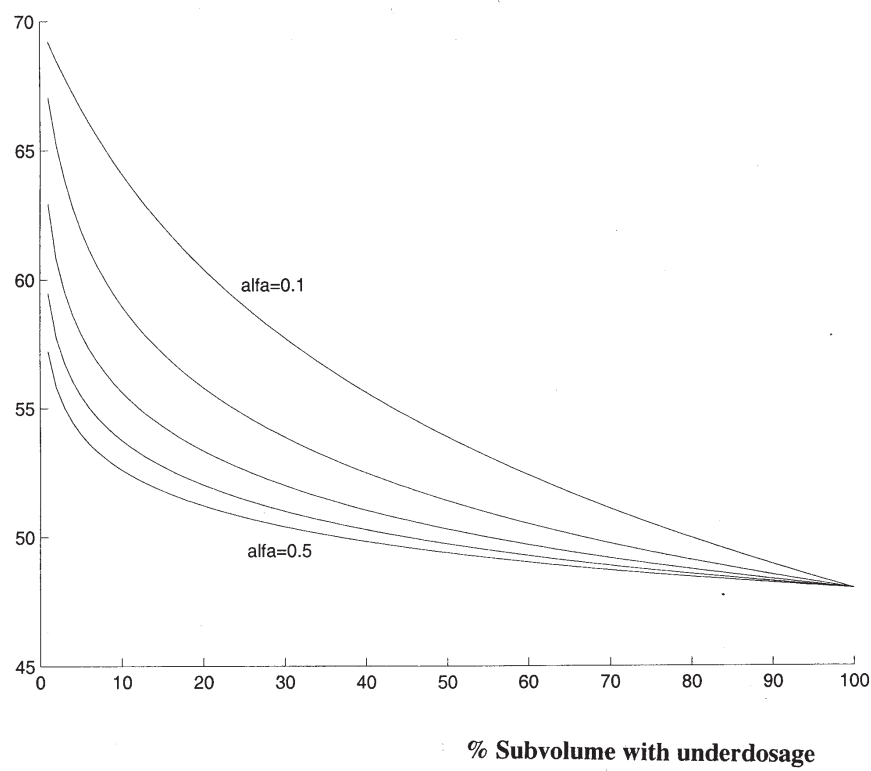

Figure 1. The under dose effect of $8 \mathrm{~Gy}$ (instead of $10 \mathrm{~Gy}$ ) if $0-100$ subvolumes. (A) The resulting DVIC-BED with different ratios $(1.5,4,10)$ with five different $\alpha$ values $(0.1-0.5)$. (B) Detail of curve with ratio $=4$.

mean (4 and 0.3 , respectively) (Fig. 2B). The curves in the middle (between the two Ds) are randomised. Although these values for ratio $\alpha$ are normal and distributed $\sim 4$ and 0.3 , the resulting curve is located lower than the mean curve (B). Thus, the effect of radio-resistant cells is amplified by the underdosage in some subvolumes.

Fig. 3 shows the effect of underdosage in subvolume in two brachytherapies, with different ratios but with the same DVIC-BED (if no miss is simulated). The DVIC-BED for no
A

DVIC-BED

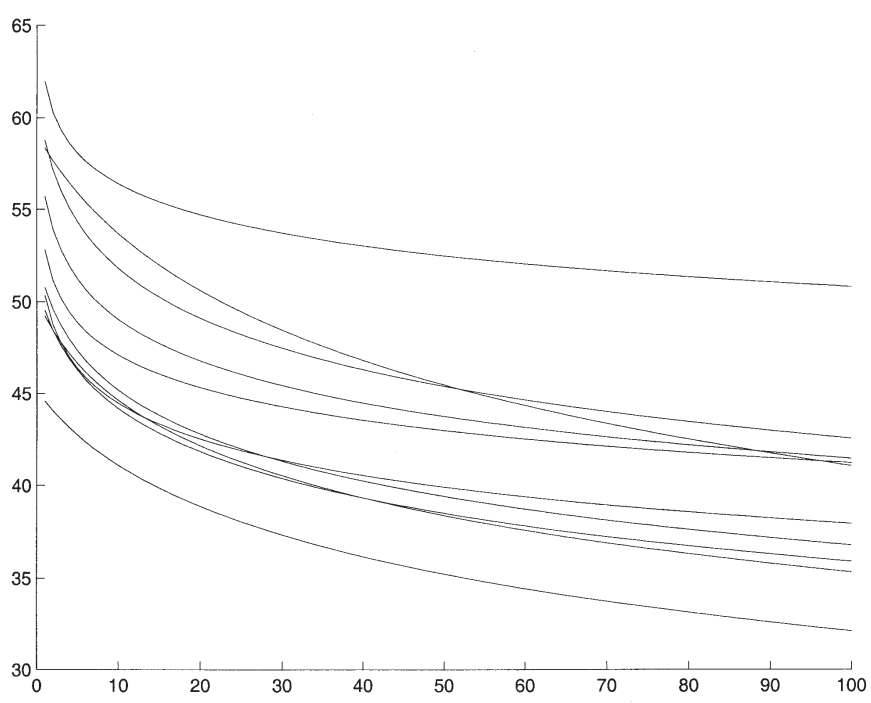

\% Subvolume with underdosage

\section{B}

\section{DVIC-BED}

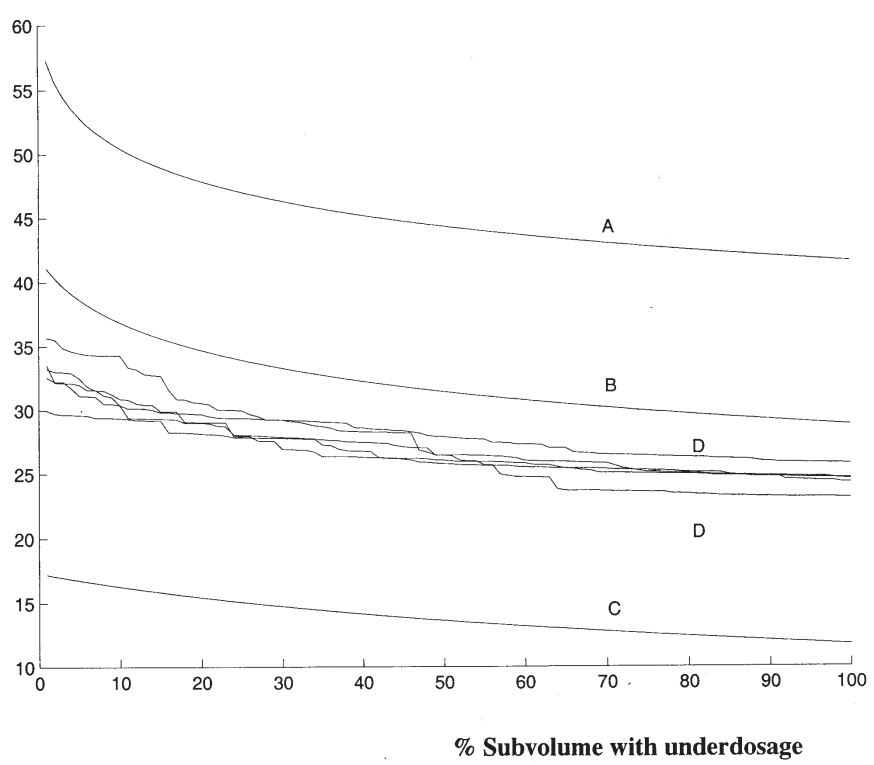

Figure 2. (A) The result of DVIC-BED on different random values (ratio and $\alpha / \beta)$ for each treatment. Same value in each subvolume of one treatment. (B) Random values for each subvolume. The highest curve (A) represents a high ratio (5) and low $\alpha(0.2)$, the lowest curve $(\mathrm{C})$ a low ratio (3) and high $\alpha(0.4)$ respectively, and (B) the mean (4 and 0.3, respectively). The curves in the middle (between the two Ds) are randomised. Although these values for ratio and $\alpha$ are normally distributed $\sim 4$ and 0.3 , the resulting curves are located lower than the mean curve (B).

miss is 153 and 150 , respectively. The treatments are both fractionated in 2 fractions with the total dose 20 Gy (ratio 1.5) and 32 Gy (ratio 4). The underdosage is $80 \%$ of the dose in $0-100 \%$ of the subvolume. The effect is the same as that shown in Figs. 1 and 2, but low ratio is more sensitive to a miss in the target volume. 


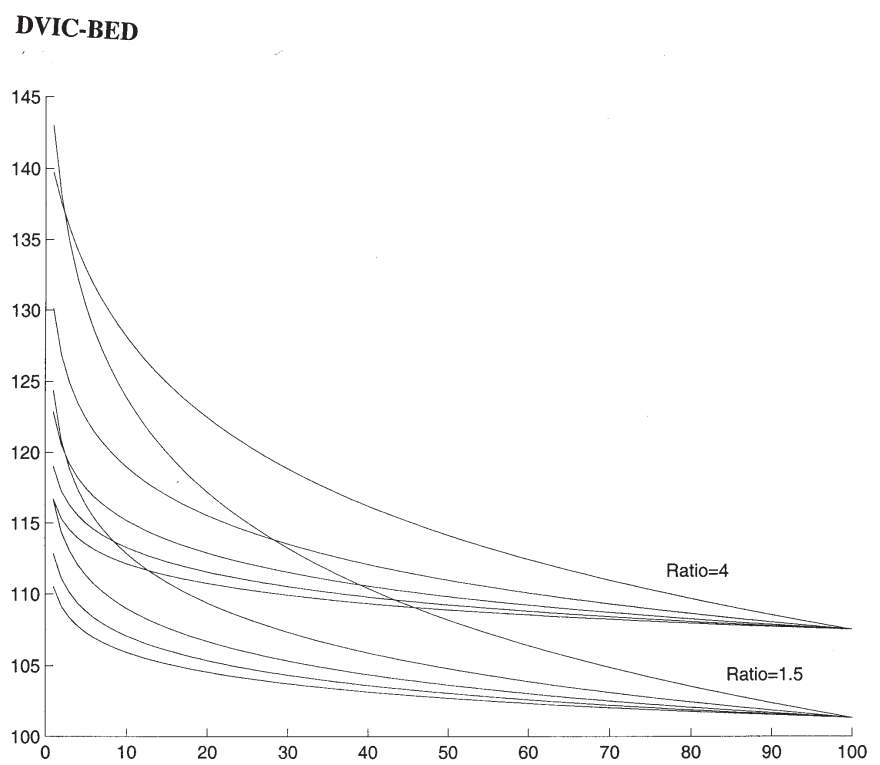

$\%$ Subvolume with underdosage

Figure 3. Comparison of two different brachytherapy schedules. DVIC-BED at different ratios, but the same DVIC-BED if no miss of the treatment volume. The treatments are both fractionated in 2 fractions with the total dose 20 Gy (ratio 1.5) and $32 \mathrm{~Gy}$ (ratio 4). The underdosage is $80 \%$ of the dose in $0-100 \%$ of the subvolume. The DVIC-BED for no miss is 153 and 150 respectively.

\section{Discussion}

It is important to note that all models are simplifications and much information concerning a tumour is still unknown. However, models help us understand and detect possible pitfalls in radiotherapy treatments, but, the interpretation of a certain result must be done carefully.

The effect of a decrease in DVIC-BED values on local control is dependent on the slope of the dose-response curve at the current dose level. The dose required for the local control of a PAC is not self-evident, nor is the shape of the doseresponse curve although Hanks among others have presented a large contribution on the issue. Higher doses seem to increase clinical and microscopic local control and Hanks and co-workers (8) have presented dose responds curves based on the pre-treatment PSA and clinical and biochemical diseasefree patients (bNED). The study clearly indicates the steep dose-response relationship in the 70-80 Gy intervals and the impact of dose escalation on bNED.

It can be estimated that a dose reduction of $5 \mathrm{~Gy}$, results in a 10\% decrease of bNED in the interval 65-70 Gy and 20-25\% in the interval 75-80 Gy using data presented by Hanks. However, dose escalation will force the BED to a flatter section of the dose-response curve, and the probability for cure will be less dependent on BED fluctuations. Therefore, an increase of the DVIC-BED far to the right part of the curve can be questioned from a clinical point of view, although this will at the same time make the treatment more resistant to a heterogeneous dose distribution.

High dose rate brachytherapy is growing in popularity for the treatment of PAC. The intention is to deliver a high dose boost to the prostate and recently monotherapy or treatments with few fractions have been presented. Data such as presented by Hanks among others are important contributions for the selection of number of fractions and total dose. However, models for dose-fraction evaluation, such as LQ and DVIC-BED, itself depends very much on the chosen radiobiological parameters, such as the $\alpha / \beta$ ratio, and this is not self-evident (see Discussion).

In this study we emphasise a possible problem with brachytherapy in general and in monotheraphy specifically - the large effect of even a small underdosage of the target. This effect is the greatest using a low $\alpha / \beta$ ratio and when $\alpha$ is high. Based on Fig. 2, one can argue that the $\alpha / \beta$ ratio is not important since even the curves with the lowest $\alpha / \beta$ ratio and the largest underdosage effect, will be better than higher $\alpha / \beta$ ratio curves. As shown in Fig. 3, the sensitivity to under dosages is present in both ratio 4 and ratio 1.5 treatments, and a low ratio is more sensitive.

If a treatment is designed with a higher $\alpha / \beta$ ratio a higher dose per fraction is required and the treatment will be less sensitive to under dosage. At the same time the treatment will be less sensitive to clones of cells with a possible lower ratio.

This study stresses the importance of including not only the desired dose given to the target, but also the dose given to all subvolumes of the target. Although a high $\alpha / \beta$ ratio will give a strong effect on the tumour, it is dangerous if this dose intensity cannot be achieved.

There is a risk using low $\alpha / \beta$ ratios, since you can design new schedules with wide therapeutic windows between the effects on the tumour versus the effect on organs at risk. However, this could be incorrect and the patient will end up receiving a sub-optimal dose. Although data indicate that the $\alpha / \beta$ ratio for PAC is low we need more reliable data based on long-term survival (not biochemical surrogate) before we use it to design new treatments. A good rule is not to use a lower $\alpha / \beta$ ratio than the $\alpha / \beta$ ratio for slow reacting tissue.

This study was designed to show how the DVIC-BED model is an extension of the BED- and LQ-model and that the DVIC-BED adds volume effect to these models. This makes DVIC-BED well suited for evaluating and reporting brachytherapy treatments.

\section{Acknowledgements}

We wish to thank Professor Jack Fowler for fruitful discussions during the preparation of the study. This study was supported by the King Gustav V Jubilee Clinic Cancer Research Foundation and Swedish Cancer Foundation, grant 060007.

\section{References}

1. Cancer statistics in Sweden 2005. Socialstyrelsen. http://www. sos.se/sos/statisti.htm

2. Martinez A, Kestin LL, Stromberg JS, Gonzalez JA, Wallace M, Gustafson GS, Edmundson GK, Spencer W and Vicini FA: Interim report of image-guided conformal high-dose-rate brachytherapy for patients with unfavorable prostate cancer: the William Beaumont Phase II dose-escalating trial. Int J Rad Oncol Biol Phys 47: 343-352, 2000.

3. Hanks GE: Radiotherapy or surgery for prostate cancer? Acta Oncol 30: 231-237, 1991.

4. Porter AT and Forman JD: Prostate Brachytherapy, an overview. Cancer 71: 953-958, 1993.

5. Hanks GE, Hanlon AL, Schultheiss TE, et al: Dose escalation with 3D conformal treatments: five year outcomes, treatment optimization, and future directions. Int J Radiat Oncol Biol Phys 41: 501-510, 1998. 
6. Lennernas B, Holmang S and Hedelin H: High-dose rate brachytherapy of prostatic adenocarcinoma in combination with external beam radiotherapy a long-term follow-up of the first 50 patients at one center. Strahlenther Onkol 178: 537-541, 2002.

7. Bucci K, Bevan A and Roach M: Advances in radiation therapy: conventional to $3 \mathrm{D}$, to IMRT, to $4 \mathrm{D}$ and beyond. Cancer J Clin 55: 117-134, 2005.

8. Lennernäs B and Nilsson S: Calculated effects of displacement errors in external beam radiotherapy of prostatic adenocarcinoma. Acta Oncol 38: 203-208, 1999.

9. Wolbarst AB: Optimization of radiation therapy II: The criticalvoxel model. Int J Radiat Oncol Biol Phys 10: 741-745, 1984.

10. Steel CG: Growth kinetics of tumours. Clarendon Press, Oxford, 1977.

11. Fowler JF: The linear-quadratic formula and progress in fractionated radiotherapy. Br J Radiol 62: 679-694, 1989.
12. King CR and Mayo CS: Is the prostate/ratio of 1.5 from Brenner \& Hall a modeling artifact? Int J Rad Oncol Biol Phys 47: 536-538, 2000.

13. Brenner DJ and Hall EJ: Fractionation and protraction for radiotherapy of prostate carcinoma. Int $\mathrm{J}$ Rad Oncol Biol Phys 43: 1095-1101, 1999.

14. Lai PP, Perez CA, Shapio SJ and Lockett MA: Carcinoma of the prostate stage $B$ and $C$ : Lack of influence of duration of radiotherapy on tumor control and treatment morbidity. Int $\mathrm{J}$ Radiat Oncol Biol Phys 19: 561-568, 1990.

15. Eklöv S, Essand M, Carlsson J and Nilsson S: Radiation sensitization by Estramustine. Studies on cultured human prostatic cancer cells. Prostate 21: 287-295, 1992.

16. DeWeese T, Dillehay LE, Shao Y and Williams JR: Low dose sensitization of human prostate cancer cells. Int J Radiat Oncol Biol Phys 30 (Suppl): 319, 1994. 